\title{
ESTUDO DE CARACTERIZAÇÃO DA DISTRIBUIÇÃO DE TAMANHO DE BOLHAS EM UMA COLUNA DE FLOTAÇÃO
}

\author{
A. S. REIS ${ }^{1}$, S. M. GONÇALVES ${ }^{1}$, F. GARDUSI ${ }^{1}$, A. M. R. FILHO ${ }^{1}$, P. H. M. LUZ ${ }^{1}$, \\ M. A. S. BARROZO \\ ${ }^{1}$ Universidade Federal de Uberlândia, Faculdade de Engenharia Química \\ E-mail para contato: sreis.angelica@gmail.com
}

\begin{abstract}
RESUMO - A principal técnica para a concentração de minérios é a flotação, processo no qual a distribuição de tamanhos das partículas e das bolhas são variáveis físicas importantes e de grande impacto, pois influem diretamente na eficiência de captura das partículas e no holdup de ar. Na flotação de partículas finas e ultrafinas o uso de microbolhas favorece a probabilidade de colisão bolha-partícula, porém, obtém resultados indesejáveis no processo devido à baixa velocidade de ascensão das microbolhas. Assim, esse trabalho teve por objetivo o estudo da formação de bolhas intermediárias (bolhas com diâmetro entre os obtidos na flotação por ar dissolvido e no sistema convencional de flotação) produzidas a partir da passagem da recirculação por um Venturi. Os resultados obtidos permitiram verificar a influência das variáveis pressão de ar, vazão de ar e pressão da linha do Venturi no holdup e na distribuição de diâmetro das bolhas que foram obtidos por técnicas de manometria e análise de imagens, respectivamente.
\end{abstract}

\section{INTRODUÇÃO}

A principal tecnologia aplicada para a concentração de minérios é o processo de flotação, o qual passou por constantes desenvolvimentos em termos de variedade e sofisticação nas últimas décadas. A flotação é um processo físico-químico utilizado para separação de partículas (ou agregados) minerais, pela adesão de bolhas de ar em uma suspensão aquosa. Dessa forma, as partículas que se aderem às bolhas de ar e formam unidades estáveis, com uma densidade aparente menor que a suspensão aquosa, ascendem (flotam) até a superfície formando uma espuma a qual é removida como concentrado. (Santos, 2010)

No processo de flotação, a distribuição de tamanho das partículas bem como o tamanho de bolhas, são variáveis físicas importantes e de grande impacto no processo, pois influem diretamente na eficiência de captura das partículas e no holdup de ar (fração de gás na mistura gás-líquido) (Rodrigues, 2004). Dessa forma, a distribuição de tamanho de partículas exige que se disponha de uma faixa de tamanho de bolhas que maximize a captura de partículas pelas bolhas, otimizando o processo de beneficiamento mineral. Por essa razão, recentemente, a caracterização destas bolhas tem atraído a atenção de muitos pesquisadores (Englert, 2008).

$\mathrm{Na}$ flotação de partículas finas e ultrafinas o uso de microbolhas favorece a probabilidade de colisão bolha-partícula, fator que influencia diretamente na eficiência da flotação. Contudo, o uso desse procedimento é limitado devido à baixa velocidade de 
ascensão das microbolhas o que leva ao arraste dos agregados obtendo resultados indesejáveis no processo (Filippov et al, 2000).

Visto que a distribuição de diâmetro de bolhas é uma variável importante no processo de flotação, a determinação precisa deste parâmetro é essencial para a compreensão dos fenômenos físicos que governam esses sistemas (Ribeiro, 2012). Diversas técnicas, invasivas e não-invasivas, tem sido utilizadas para a determinação da distribuição de diâmetro de bolhas, sendo a técnica de análise de imagens a mais utilizada para sistemas bifásicos (gáslíquido) e trifásicos (gás-líquido-sólido) (Englert, 2008).

Sendo assim, o objetivo deste trabalho foi promover a geração de bolhas intermediárias através da passagem forçada da corrente de recirculação por um Venturi e a caracterização da formação destas bolhas a partir da avaliação da influência da pressão de ar, vazão do rotâmetro e pressão da linha do Venturi no holdup e na distribuição de diâmetro das bolhas.

\section{MATERIAIS E MÉTODOS}

\subsection{Unidade Experimental}

A unidade experimental, representada pela Figura 1, encontra-se no Laboratório de Sistemas Particulados da Faculdade de Engenharia Química da Universidade Federal de Uberlândia. A unidade consiste de uma coluna de flotação feita em acrílico composta uma seção cilíndrica com $150 \mathrm{~cm}$ de comprimento e $4 \mathrm{~cm}$ de diâmetro, abaixo uma seção troncocônica com $9,5 \mathrm{~cm}$ de altura e abaixo desta outra seção cilíndrica com $12 \mathrm{~cm}$ de altura e 10 $\mathrm{cm}$ de diâmetro.

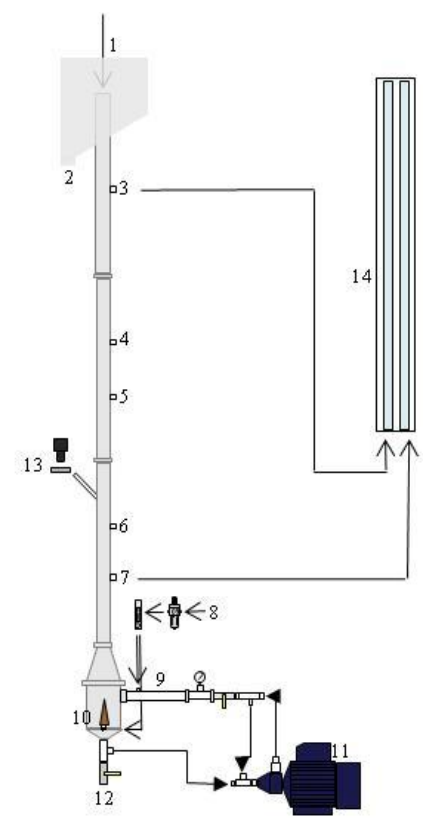

Legenda:

1- alimentação de água

2 - ponto de coleta do concentrado

$3,4,5,6,7$ - pontos de recirculação e amostragem do holdup

8 - sistema de alimentação de ar comprimido com filtro de ar e rotâmetro

$9-$ Venturi

10 - aerador

11 - bomba peristáltica

12 - ponto de coleta do rejeito

13 - sistema de amostragem de bolhas

14 - manômetros diferenciais

Figura 1 - Unidade Experimental.

A coluna de flotação foi construída de forma que fosse possível a geração de bolhas através de duas diferentes técnicas: a geração de bolhas com o sistema operando com ar disperso, no qual a alimentação de ar é feita diretamente no aerador (10), e também, a geração 
de bolhas pela passagem forçada da corrente de recirculação por um Venturi (9), com restrição de $2 \mathrm{~mm}$ de diâmetro, no qual o ar é aspirado e as bolhas são geradas.

\subsection{Metodologia Experimental}

Metodologia para medida de holdup: Os valores dos holdup da coluna foram obtidos a partir dos manômetros diferenciais (14) acoplados à coluna como ilustrados na Figura 1. Assim a partir dos valores de pressão obtidos foi possível obter o valor de holdup (Dobby e Finch, 1990). Os desníveis observados nos manômetros eram coletados no momento em que não se observava uma variação significativa no holdup da coluna.

Metodologia para medida do diâmetro de bolhas: O método consistia num sistema de amostragem de bolhas o qual era composto de um amostrador projetado por Ribeiro (2012), uma câmera de alta velocidade e um estereomicroscópio. A amostragem era feita através de um tubo de aço, conectado ao amostrador, com $6 \mathrm{~cm}$ de diâmetro e $10 \mathrm{~cm}$ de comprimento posicionado no centro e com um ângulo de $45^{\circ}$ com relação à coluna. Com o auxílio de uma bomba peristáltica, posicionada a jusante do amostrador, as bolhas eram succionadas do interior da coluna com velocidade previamente controlada de forma a diminuir o efeito de quebra e coalescência das bolhas coletadas. As imagens obtidas foram capturadas diretamente no computador e a análise das imagens foi obtida através do software ImageJ, o qual, a partir de uma calibração prévia, fornecia a área das bolhas filmadas e o diâmetro de bolhas calculado foi o diâmetro da esfera de mesma área projetada. A partir da distribuição de diâmetros obtida para cada condição testada foi possível obter o diâmetro médio aritmético $\left(\mathrm{d}_{\mathrm{m}}\right)$ e o diâmetro médio de Sauter $\left(\mathrm{d}_{32}\right)$ a partir das Equações 1 e 2, respectivamente.

$$
\begin{aligned}
& d_{m}=\frac{\sum d_{i}}{n} \\
& d_{32}=\frac{\sum d_{i}^{3}}{\sum d_{i}^{2}}
\end{aligned}
$$

Sendo, $\mathrm{d}_{\mathrm{i}}$ é o diâmetro individual de bolha medido e $\mathrm{n}$ a quantidade total de bolhas medidas.

Com o objetivo de verificar os resultados obtidos, as bolhas foram medidas por um segundo método constituído pela filmagem direta da coluna em operação, com a mesma câmera de alta velocidade. O tratamento das imagens foi conduzido da mesma forma que no método anterior e a deformação nelas provocada pelo acrílico foi corrigida por meio de uma filmagem com esferas de diâmetro conhecido, colocadas no interior da coluna em operação.

\subsection{Planejamento Experimental}

Para caracterização da distribuição de bolhas geradas foi proposto um PCC (Planejamento Composto Central), de forma a avaliar a influência de três variáveis independentes: pressão da linha de ar comprimido (Par), vazão de ar no rotâmetro (Qrot) e a pressão da corrente de alimentação do Venturi proveniente da passagem da recirculação por uma bomba peristáltica $(\mathrm{Pb})$. As variáveis respostas analisadas foram o diâmetro médio de 
bolhas $\left(\mathrm{d}_{\mathrm{m}}[\mu \mathrm{m}]\right)$, diâmetro médio de Sauter $\left(\mathrm{d}_{32}[\mu \mathrm{m}]\right)$ e o holdup $\left(\varepsilon_{\mathrm{o}}[-]\right)$. A fim de manter a ortogonalidade do sistema foi utilizado $\alpha=1,287$. Na Tabela 1 encontram-se os valores correspondentes a cada nível utilizado nos testes do PCC.

\begin{tabular}{c|ccc}
\multicolumn{5}{c}{ Tabela 1 - Planejamento Composto Central (PCC) } \\
\hline Teste & Par(bar) & Qrot(L/h) & $\boldsymbol{P b ( b a r )}$ \\
\hline 1 & 2.0 & 5.0 & 2.0 \\
2 & 2.0 & 5.0 & 4.0 \\
3 & 2.0 & 20.0 & 2.0 \\
4 & 2.0 & 20.0 & 4.0 \\
5 & 4.0 & 5.0 & 2.0 \\
6 & 4.0 & 5.0 & 4.0 \\
7 & 4.0 & 20.0 & 2.0 \\
8 & 4.0 & 20.0 & 4.0 \\
9 & 1.7 & 12.5 & 3.0 \\
10 & 4.3 & 12.5 & 3.0 \\
11 & 3.0 & 3.0 & 3.0 \\
12 & 3.0 & 22.0 & 3.0 \\
13 & 3.0 & 12.5 & 1.7 \\
14 & 3.0 & 12.5 & 4.3 \\
15 & 3.0 & 12.5 & 3.0 \\
16 & 3.0 & 12.5 & 3.0 \\
\hline
\end{tabular}

\section{RESULTADOS E DISCUSSÕES}

\subsection{Distribuição de Diâmetro de Bolha}

Seguindo o objetivo desse trabalho, a geração de bolhas intermediárias foi comprovada pela análise da distribuição de tamanhos obtidas em três diferentes sistemas para geração de bolhas: flotação por ar dissolvido - FAD, no qual microbolhas são geradas pela descompressão súbita de uma mistura saturada de ar-água através da passagem desta por válvula agulha; ar disperso, em que as bolhas são geradas a partir da injeção de ar em uma matriz porosa e; bolhas geradas pela passagem forçada de uma corrente de recirculação, juntamente com ar injetado, por um Venturi (bolhas de tamanho intermediário).

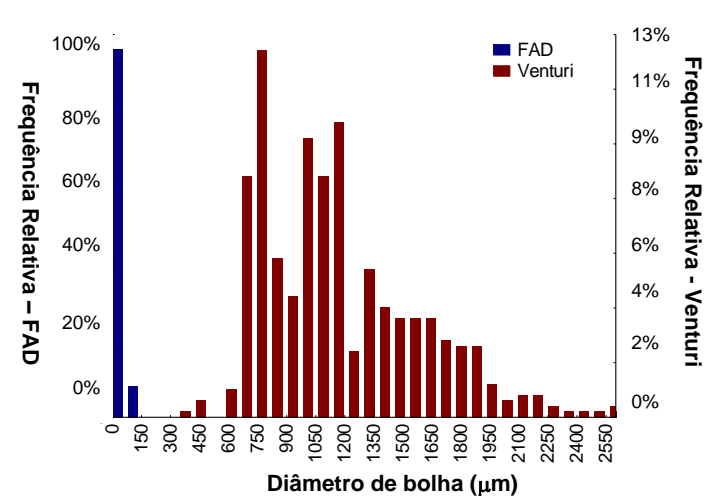

(a)

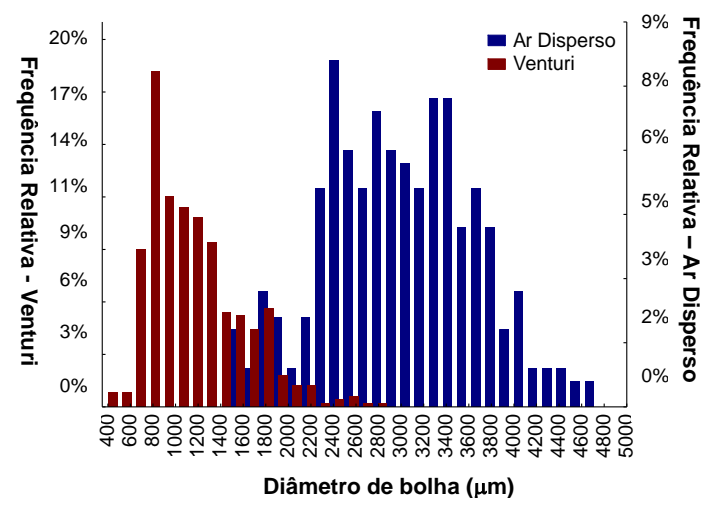

(b)

Figura 2 - Histogramas comparativos da distribuição de bolhas geradas pelo Venturi com as geradas pela FAD (a) e por ar disperso (b). 
Os resultados mostrados na Figura 2 (a) e (b) comprovam a eficiência do sistema operando com o Venturi na formação de bolhas intermediárias, uma vez que a distribuição de diâmetros de bolhas obtida para o sistema operando com o Venturi foi maior que a distribuição de diâmetro de bolhas obtida com a FAD (Figuara 2a) e menor que o sistema operando com ar disperso (Figura 2b). Os dados de distribuição de diâmetro de bolhas obtidos pela FAD são referentes ao trabalho publicado por Santana et al. (2012).

Esses resultados são coerentes com a literatura científica que mostra que as microbolhas, geradas pela $\mathrm{FAD}$, apresentam diâmetro menor que $100 \mu \mathrm{m}$, as bolhas intermediárias se encontram na faixa de 200 a $1400 \mu \mathrm{m}$ e as bolhas geradas por ar disperso na faixa de 600 a $2000 \mu \mathrm{m}$ (Rodrigues, 2004).

\subsection{Efeito das variáveis independentes}

$\mathrm{Na}$ análise de regressão do PCC, as variáveis independentes pressão da linha de ar comprimido (Par), vazão de ar medida no rotâmetro (Qar) e a pressão da corrente de alimentação do Venturi $(\mathrm{Pb})$ foram adimensionalizadas conforme as Equações 3, 4 e 5, respectivamente.

$$
\begin{aligned}
X 1 & =\frac{(\text { Par }-3 b a r)}{1 b a r} \\
X 2 & =\frac{(\text { Qrot }-12,5 L / h)}{7,5 L / h} \\
X 3 & =\frac{(P b-3 b a r)}{1 b a r}
\end{aligned}
$$

Para cada resposta avaliada foram obtidas, a partir de regressões múltiplas, equações empíricas que demonstram quais e de que forma as variáveis independentes influenciam o sistema. A Equação 6 foi obtida para medição do valor de holdup $\left(\hat{y}_{1}\right) \operatorname{com} \mathrm{r}^{2}=0,987$. A Equação 7 , representa a influência de cada variável no diâmetro médio de Sauter $\left(\hat{y}_{2}\right)$ e foi obtido um coeficiente de correlação quadrático no valor de 0,953 . O diâmetro médio de bolhas $\left(\hat{y}_{3}\right)$ teve como modelo a expressão apresentada na Equação 8 com um $\mathrm{r}^{2}=0,942$.

$$
\hat{y}_{1}=1,505+\underline{\boldsymbol{x}^{\prime}} \underline{\boldsymbol{b}_{1}}+\underline{\boldsymbol{x}^{\prime}} \underline{\boldsymbol{B}_{\mathbf{1}}} \underline{\boldsymbol{x}}
$$

em que:

$$
\begin{aligned}
& \underline{b_{1}}=\left[\begin{array}{c}
-0,084 \\
0,981 \\
0,131
\end{array}\right] \text { e } \underline{B_{1}}=\left[\begin{array}{ccc}
0 & 0,103 & 0 \\
0 & 0 & 0,178 \\
0 & 0 & 0
\end{array}\right] \underline{x}=\left[\begin{array}{l}
x_{1} \\
x_{2} \\
x_{3}
\end{array}\right] \\
& \hat{y}_{2}=2612,121+\underline{\boldsymbol{x}^{\prime}} \underline{\boldsymbol{b}_{1}}+\underline{\boldsymbol{x}} \underline{\boldsymbol{B}_{1}} \underline{\boldsymbol{x}}
\end{aligned}
$$

em que:

$$
\underline{b_{1}}=\left[\begin{array}{c}
-8,638 \\
542,814 \\
-110,858
\end{array}\right] \text { e } \underline{B_{1}}=\left[\begin{array}{ccc}
0 & -228,219 & 0 \\
0 & -428,875 & 0 \\
0 & 0 & 0
\end{array}\right] \underline{x}=\left[\begin{array}{l}
x_{1} \\
x_{2} \\
x_{3}
\end{array}\right]
$$




$$
\hat{y}_{3}=2185,851+\underline{\boldsymbol{x}^{\prime}} \underline{\boldsymbol{b}_{\mathbf{1}}}+\underline{\boldsymbol{x}^{\prime}} \underline{\boldsymbol{B}_{\mathbf{1}}} \underline{\boldsymbol{x}}
$$

em que:

$$
\underline{b_{1}}=\left[\begin{array}{c}
-102,725 \\
793,611 \\
0
\end{array}\right] \text { e } \underline{B_{1}}=\left[\begin{array}{ccc}
0 & -146,110 & 0 \\
0 & -589,738 & 0 \\
0 & 0 & 0
\end{array}\right] \underline{x}=\left[\begin{array}{l}
x_{1} \\
x_{2} \\
x_{3}
\end{array}\right]
$$

Na análise dos modelos empíricos obtidos notou-se que a vazão de ar (X2) apresentou maior efeito significativo nas três respostas, sendo que o aumento desta variável provoca um aumento nas três respostas analisadas. Para o diâmetro médio, a pressão conferida ao sistema pela bomba na passagem forçada da corrente de recirculação pelo Venturi (X3) não apresentou efeito significativo para as análises estatísticas realizadas com um nível de significância de $10 \%$.

As Figuras 3 e 4 mostram a influência das variáveis vazão de ar (Qrot) e pressão do ar (Par) para uma pressão na linha do Venturi $(\mathrm{Pb})$ no valor de 3 bar (nível zero para a variável adimensionalizada), no $\varepsilon_{\mathrm{o}}$ e no $\mathrm{d}_{\mathrm{m}}$.

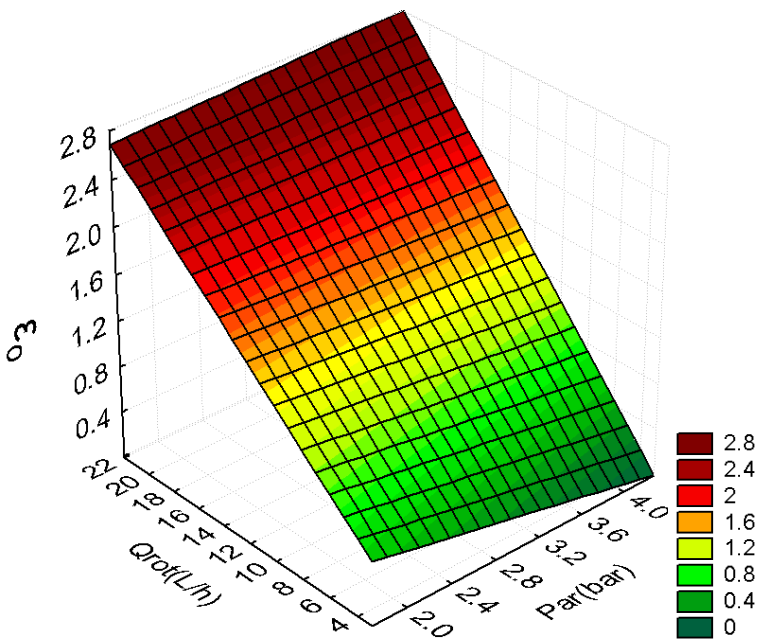

Figura 3 - Superfície de resposta para o $\varepsilon_{0}$ em função da pressão do ar (Par) e da vazão no rotâmetro (Qrot) com a pressão da linha do Venturi no ponto central igual a 3 bar.

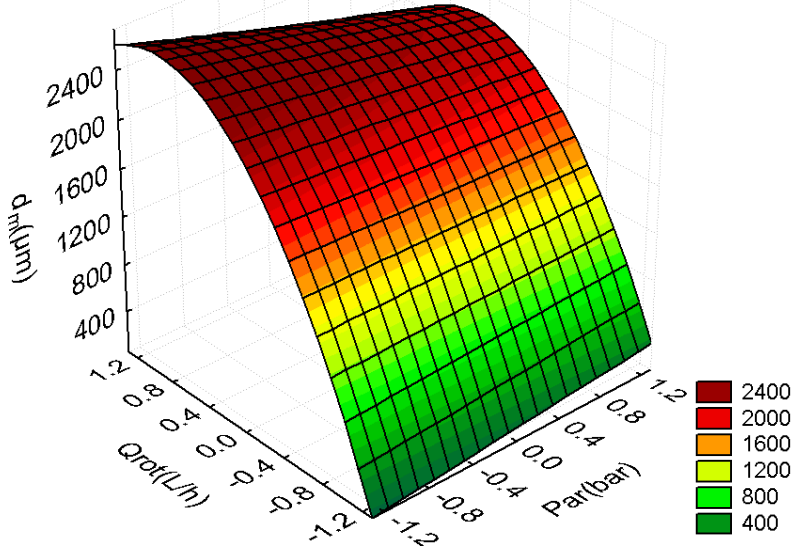

Figura 4 - Superfície de resposta para o $d_{m}$ em função da pressão do ar (Par) e da vazão no rotâmetro (Qrot) com a pressão da linha do Venturi no ponto central igual a 3 bar.

Nota-se que para baixos valores de vazão de ar a diminuição da pressão da linha de ar resultou no aumento do valor de $\varepsilon_{o}$ e na diminuição do diâmetro de bolha. À medida que se aumenta a vazão do ar observa-se uma inversão no sistema e o diâmetro de bolha aumenta com a diminuição da pressão do ar, enquanto o holdup sofre um leve decaimento no seu valor. Esse fenômeno se deve provavelmente ao processo de quebra e coalescência que ocorre na passagem das bolhas pelo Venturi indicando que em vazões de ar mais baixas o aumento da pressão do ar favorece a coalescência das bolhas no Venturi, todavia, quando se aumenta a vazão de ar concomitantemente com a pressão o fenômeno que é favorecido é o de quebra e as bolhas tem seu diâmetro médio diminuído. Foi observada a mesma tendência obtida no $\mathrm{d}_{\mathrm{m}}$ para o d $\mathrm{d}_{32}$. 
Segundo Finch et al. (2012) o tamanho de bolha e holdup apresentam-se sempre de forma inversa que pode ser explicado pela relação da velocidade de ascensão da bolha com o seu diâmetro, dessa forma, à medida que o diâmetro de bolha aumenta, maior é sua velocidade de ascensão e menor seu tempo de retenção no interior da coluna causando uma redução no valor do holdup.

Mantendo a variável Par(pressão do ar) no seu ponto central, observamos que o aumento na pressão na linha do Venturi ( $\mathrm{Pb}$ ), para altas vazões de ar (Qrot), culminou no aumento do $\varepsilon_{\mathrm{o}}$ e na diminuição no $\mathrm{d}_{32}$, conforme esperado. Para baixas vazões de ar, o aumento de $\mathrm{Pb}$ resultou na diminuição do $\mathrm{d}_{32}$, porém apresentou pouca influência no holdup, levando a uma leve diminuição do seu valor, conforme indicado nas Figuras 5 e 6 . Nesse caso, a pressão da linha do Venturi, apesar de sua baixa influência no sistema, mostra que provavelmente o aumento da pressão provocada pela bomba na linha do Venturi favorece o processo de quebra das bolhas tendo como consequiência a diminuição do seu diâmetro médio.

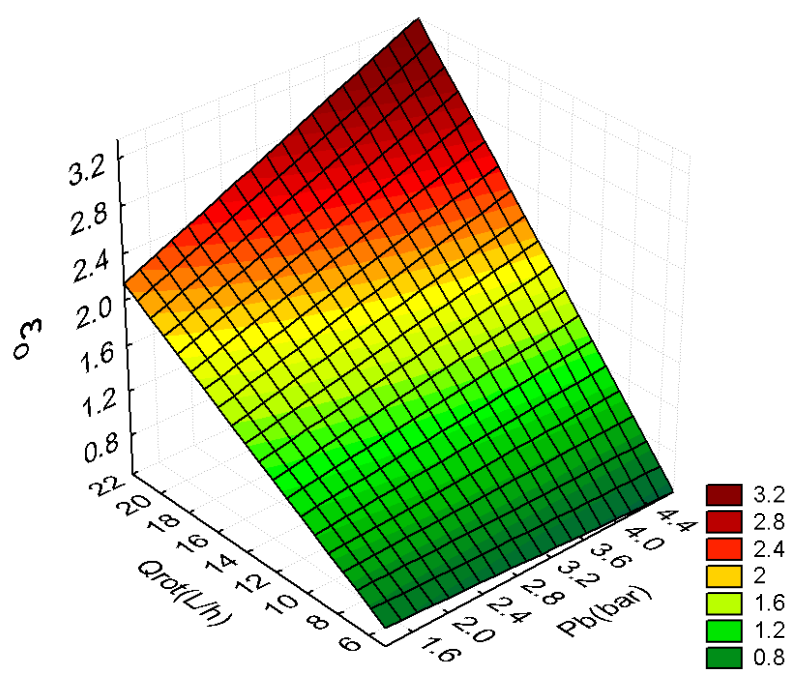

Figura 5 - Superfície de resposta para o $\varepsilon_{0}$ em função da vazão no rotâmetro (Qrot) e da pressão na linha do Venturi $(\mathrm{Pb})$ e com a pressão do ar no ponto central igual a 3 bar.

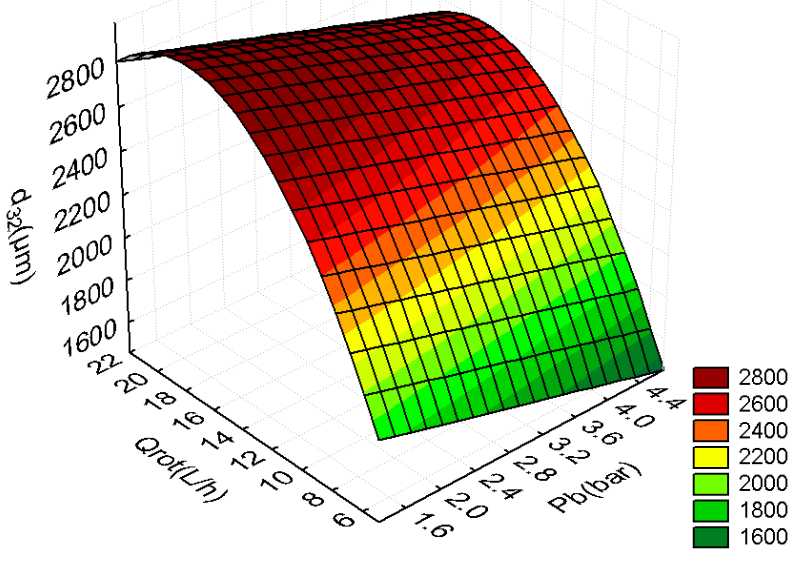

Figura 6 - Superfície de resposta para o $\mathrm{d}_{32}$ em função da vazão no rotâmetro (Qrot) e da pressão na linha do Venturi $(\mathrm{Pb})$ e com a pressão do ar no ponto central igual a 3 bar.

De maneira geral, observou-se que, nas condições estudadas, as regiões correspondentes à faixa de diâmetro de bolhas intermediárias apresentaram baixos valores para o holdup devido à baixa vazão de ar, ou seja, a quantidade de ar alimentada ao sistema é pequena, por isso, apresentando um menor diâmetro de bolha.

\section{CONCLUSÕES}

Através da análise de imagens foi possível mensurar o diâmetro das bolhas geradas no interior da coluna de flotação e constatar que as bolhas geradas pela passagem forçada da corrente de recirculação pelo Venturi acoplado à coluna de flotação são bolhas com tamanho intermediário, de acordo com o objetivo proposto. A geração deste tipo de distribuição de 
bolhas é fundamental para a eficiência do processo de flotação. A variável mais influente no processo foi a vazão de ar, conforme identificado pelos resultados obtidos no PCC. O diâmetro de bolha e o holdup apresentaram comportamento inverso, o que está de acordo com o observado na literatura.

\section{AGRADECIMENTOS}

Agradecemos ao CNPQ e a Fundação de Amparo à Pesquisa do Estado de Minas Gerais (FAPEMIG) pelo apoio Financeiro para a realização da pesquisa e ainda a FAPEMIG pelos recursos concedidos no Projeto de Participação Coletiva em Eventos Técnicos-Científicos (PCE-00082-14)

\section{REFERÊNCIAS}

ENGLERT, A. H. Flotação por ar dissolvido (FAD) de micropartículas, caracterização de microbolhas e medidas de força de interação bolha-partícula. Tese de Doutorado, PPGEM - UFRGS, 2008.

DOBBY, G.S.; FINCH, J.A. Column flotation. Pergamon Press. 1990.

FILIPPOV, L. O.; JOUSSEMET, R.; HOUOT, R. Bubble Spargers in Column Flotation: Adaptation to precipitate flotation. Miner. Eng., v 13, p 37-51, 2000.

FINCH, J. A. ; ZHANG, W. ; ZHOU, X. Determining independent control of dual-frother systems - Gas holdup, bubble size and water overflow rate. Mine. Eng., v. 39, p. 106116, 2012.

GUOYING, Z.; HONG, Z.; NING, X. Flotation bubble image segmentation based on seed region boundary growing. Mii. Sci. and Tech, v. 21, p. 239-242, 2011.

RIBEIRO, J. A. Contribuição ao estudo experimental e em CFD da fluidodinâmica de colunas de bolhas com aplicações na separação de misturas oleosas e processamento mineral por ar dissolvido, Tese de Doutorado, UFU, Uberlândia (MG), 2012.

RODRIGUES, R. T. Desenvolvimento da técnica LTM-BSizer para a caracterização de bolhas e avaliação de parâmetros no processo de flotação. Dissertação de doutorado, UFRGS, Porto Alegre (RS), 2004.

RUBIO, J.; GARIBAY, R. P.; RAMOS, E. M. Gás dispersion measurements in microbubble flotation systems. Miner. Eng., v. 26, p. 34-40, 2012.

SANTANA, R. C.; RIBEIRO, J. A., SANTOS, M. A.; REIS, A. S.; ATAÍDE, C. H.; BARROZO, M. A. S. Flotation of fine apatitic ore using microbubbles. Sep. And Purif, Technol., v. 98. p. 402-409, 2012.

SANTOS, M. A. Estudo da influência de íons contaminantes na flotação de apatita em coluna, Dissertação de Mestrado, UFU, Uberlândia (MG), 2010. 